\title{
1-Y-E1-1 YIA
}

Applicability of @WQS@NORD-1,@WQE@ a light-reactive NO releaser, to the lower urinary tract dysfunction

Kotomi Maeda ${ }^{1}$, Yuji Hotta ${ }^{1}$, Naoya leda $^{2}$, Tomoya Kataoka ${ }^{3}$, Hidehiko Nakagawa ${ }^{2}$, Kazunori Kimura ${ }^{1,3}$

${ }^{I}$ Dept. Hospital Pharm., Grad. Sch. Pharm. Sci., Nagoya City Univ., ${ }^{2}$ Dept. Organic \& Medicinal Chem., Grad. Sch. Pharm. Sci., Nagoya City Univ., ' Dept. Clinical Pharm., Grad. Sch. Med. Sci., Nagoya City Univ. 